\title{
INESTABILIDAD POLÍTICA Y CAÍDAS PRESIDENCIALES EN SUDAMÉRICA: CAUSAS Y CONSECUENCIAS
}

\author{
Andre Coelho \\ andreluizrj@gmail.com \\ Universidade Federal do Estado do Rio de Janeiro (UNIRIO) \\ Instituto Superior La Salle RJ.
}

\begin{abstract}
Tomando como variable la actuación de los movimientos sociales y de la sociedad civil en las crisis presidenciales en los últimos 20 años en Argentina, Brasil, Bolivia y Ecuador, el artículo analiza cómo la conformación de las instituciones políticas influyó directamente en el desarrollo de las crisis políticas y en la caída de presidentes. Para ello, se realiza una revisión crítica de la literatura destacando los diversos enfoques existentes, sus puntos de encuentro y divergencia.
\end{abstract}

Palabras clave: Presidentes, sociedad civil, inestabilidad, movimientos sociales.

\section{POLITICAL INSTABILITY AND PRESIDENTIAL OUSTER IN South America: Causes and Consequences}

Taking the behaviour of social movements and civil society during presidential crises during the last twenty years in Argentina, Brazil, Bolivia and Ecuador as a variable, the article analyzes how the design of political institutions impacted on the development of political crises and presidential downfall. To do so, a critical literature review is carried out, highlighting the various existing approaches, their commonalities and differences.

Keywords: Presidents, civil society, instability, social movements. 


\section{INTRODUCCIÓN ${ }^{1}$}

Este artículo tiene como objetivo presentar una comparación entre la ocurrencia de crisis políticas y la estabilidad presidencial en Argentina, Brasil, Bolivia y Ecuador en el periodo inmediatamente posterior a la redemocratización e implementación de las reformas estructurales. Para ello, se discute las contribuciones contemporáneas sobre el tema, analizando sus principales puntos de convergencia y distanciamiento, además de sus distintos enfoques. De ese modo, se considera principalmente la influencia del diseño institucional del sistema político y su desempeño efectivo, además de la actuación de los movimientos sociales y de la sociedad civil con el propósito de comprender cuáles fueron los principales factores que propiciaron, o no, sucesivas caídas del jefe del Ejecutivo.

Los países analizados poseen en común una herencia histórica de subdesarrollo e inestabilidad a lo largo del siglo XX, con oscilaciones entre periodos democráticos y autoritarios. Con el advenimiento de la democracia (en 1979 en Ecuador, 1982 en Bolivia, 1983 en Argentina y 1985 en Brasil) y la implementación de las reformas de mercado y de Estado, nuevas directrices tomaron la agenda pública, motivadas por cambios significativos en los escenarios externo e interno. Autores como Anastasia, Ranulfo y Santos sustentan que "a lo largo de la accidentada trayectoria de la reconstrucción de la democracia, los países de Sudamérica pasaron por procesos simultáneos de diversificación socioeconómica y cultural (...) y de manutención y/o profundización de patrones de pobreza y desigualdad social" (Anastasia, Ranulfo y Santos, 2004: 8).

Siendo así, y considerando sus particulares, los respectivos países poseen significativas similitudes, sobre todo al considerar factores estructurales como dependencia económica, pobreza, desigualdad y principalmente la historia reciente de crisis políticas y económicas, además de las ya citadas caídas presidenciales.

Además, la selección de los países se explica primordialmente por la diversidad de situaciones relativas a la estabilidad de los mandatarios. En ese sentido, uno de los aspectos más relevantes de los casos analizados reside en el hecho de que todos los presidentes que dejaron el poder desde 1989 -incluyendo los que cayeron antes del tiempo previsto- fueron substituidos por nuevos presidentes civiles. Esa observación demuestra que, a pesar de todos los problemas enfrentados por la democracia en el

Una versión preliminar de este trabajo fue presentada en el XXI Congreso Mundial de Ciencia Política, ¿Descontento Global? Dilemas de cambio, Santiago de Chile, 12 al 16 de julio de 2009. 
periodo, no volvieron las soluciones autoritarias de décadas anteriores. Los cambios de Jefe del Ejecutivo fueron cambios al interior de los regímenes y no rupturas de los mismos. Obras recientes como la de Pérez Liñán (2008, 2009), Llanos y Marsteintredet (2010), Samuels y Hochstetler (2011), llaman la atención sobre la paradoja de las sucesivas caídas presidenciales en los países de Sudamérica independientemente del tipo de régimen o de la emergencia de democracias estables con gobiernos inestables.

Se pretende demostrar que la actuación de la sociedad civil y de los movimientos sociales organizados fue fundamental para la manutención y la caída de los presidentes en el periodo. Contrariamente a lo que una mirada normativista podría comprender, la acción de esos actores no debe ser percibida como algo perjudicial para la democracia, sino como una especie de rendición de cuentas popular en la búsqueda de una mayor correspondencia entre los deseos de los ciudadanos y la efectiva actuación de sus representantes. Siendo así, apunto que en algunos casos la gran inestabilidad política e incluso el desmoronamiento de los mandatos presidenciales en los países analizados ha servido como una especie de reinicio para un sistema político que se mostraba muy problemático e incapaz de resolver los conflictos de la sociedad. Tales eventos, al ser percibidos más allá de una clave analítica formal, pueden ser vistos como inspiradores para la formulación de un nuevo modelo de democracia que se presenta de forma necesariamente más incluyente y participativa (al menos en lo que se refiere a los nuevos textos constitucionales puestos en práctica en algunos de de esos países), principalmente cuando tomamos en cuenta la herencia de tantos años de autoritarismo y severas crisis económicas.

\section{CRISIS POLÍTICAS Y CAÍDAS PRESIDENCIALES EN LA AMÉRICA LATINA CONTEMPORÁNEA}

La ocurrencia de frecuentes crisis políticas y económicas, de caídas del Ejecutivo, de las diferentes respuestas del sistema político y de la actuación de la sociedad civil organizada a través de los movimientos sociales, nos lleva a la necesidad de una profundización teórica sobre los márgenes de libertad de acción de los mandatarios de los países en cuestión. Si en el pasado reciente la inestabilidad política y presidencial de la región ocurría a través de golpes militares, hoy transcurre de acuerdo con la dinámica del juego democrático, con amplia participación de las masas y de las instituciones representativas. 
Hochstetler (2007:11) afirma que 23\% de los presidentes latinoamericanos electos democráticamente desde la década de 1970 fueron forzados a dejar sus cargos antes del término de sus mandatos. La autora llama la atención sobre la importancia de esos números y para la poca atención sistemática que reciben las caídas presidenciales en los debates sobre la calidad de la democracia y la inestabilidad de los sistemas presidenciales. De los 40 presidentes electos en la región entre 1970 y 2003, 16 de ellos (40\%) enfrentaron desafios a su permanencia en el cargo y nueve (23\%) tuvieron que concluir prematuramente sus mandatos "fijos". Así, los presidentes latinoamericanos no pueden asumir que podrán ejercer un mandato fijo y determinado. Del mismo modo, Hochstetler sugiere que las elecciones presidenciales directas en Sudamérica no dan a los presidentes una legitimidad consistente que dure todo el periodo de su mandato constitucional.

Pérez Liñán (2008:106), en un artículo que analiza América Latina en el periodo comprendido entre 1985 y 1995 llega a resultados semejantes, afirmando que 13 de los presidentes electos fueron removidos de sus cargos o forzados a renunciar, cuenta que se eleva a 18 mandatarios si incluimos a los vicepresidentes o los sucesores nombrados para completar los mandatos de aquellos que tampoco lograron concluir los suyos o convocaron elecciones anticipadas. Sin embargo, el autor concluye que tales acontecimientos no constituyen un fenómeno pasajero que se restringe a cierto contexto histórico, ya que ocurrieron en contextos institucionales diferentes.

Para Samuels y Hochstetler (2011:141) desde 1978 América Latina fue el escenario de una ola de desafios a los presidentes, siendo que 16 de ellos interrumpieron sus mandatos prematuramente. No obstante, afirman que las consecuencias de este nuevo tipo de inestabilidad son generalmente mucho más supuestas que efectivamente analizadas con criterio por la literatura. Los autores afirman que no existe ninguna evidencia de quiebra sistemática de legitimidad, interrupción en la gobernanza o erosión del presidencialismo como tipo de régimen. Para Samuels y Hochstetler, la historia reciente de resolución pacífica de crisis presidenciales muestra la resiliencia de la democracia en la América Latina contemporánea.

Uno de los aspectos relevantes para escoger los casos reside en el hecho de que Ecuador y Bolivia presentan trayectorias semejantes tanto en relación con las sucesivas caídas presidenciales como en la forma peculiar en que ocurrieron y los actores involucrados en el proceso. El caso argentino también se parece a los anteriores, considerando la grave crisis política y económica que atravesó el país en el inicio de los años 2000, provocando quiebras institucionales y renuncia de presidentes. Mientras tanto, Brasil se distancia de los otros países por la ocurrencia de un caso de juicio 
político al presidente ${ }^{2}$ justo después del retorno a la democracia y por la posterior estabilidad presidencial que perdura hasta nuestros días.

\section{Período analizado}

El periodo analizado es el inmediatamente posterior al retorno de la democracia y de la implementación de las reformas de mercado. Dos son los principales motivos para escoger el de 1989 como el punto de partida para la investigación: el evento paradigmático de la caída del muro de Berlín, asociado con la creación del Consenso de Washington; además de razones de orden práctico, por representar el año del comienzo de un nuevo ciclo de presidentes electos democráticamente en los cuatro países considerados.

En Argentina, se analizan los dos gobiernos de Carlos Menem (1990-1995; 19951999), substituido por Fernando de la Rúa (1999), que dejó el cargo por intensas movilizaciones populares en 2001, cuando después de una gran crisis decisoria el Congreso escogió a Eduardo Duhalde como presidente interino. En 2003, Néstor Kirchner fue electo nuevo presidente de Argentina y dejó el poder en 2007, substituido por su esposa Cristina Fernández, reelecta en 2011.

En Brasil, el periodo inicia con el gobierno de Collor (1990-1992), depuesto por el Congreso mediante un juicio político (1992), cuando Itamar Franco (1992-1994), el vicepresidente, asumió el gobierno. Fernando Henrique Cardoso, que había sido ministro de Hacienda en la gestión anterior, inició su primer mandato (1995-1998) y fue posteriormente reelecto (1999-2002). En 2003, Luiz Inácio Lula da Silva asume la presidencia y logró también la reelección en 2006. Finalmente, en 2010, Dilma Roussef fue electa la primera presidente del país.

En Bolivia, el estudio abarca el primer gobierno de Sánchez de Lozada (1993-1997) y el mandato de Hugo Banzer (1996-2001), cuando dejó el poder por causas de salud. Banzer fue substituido por el gobierno de transición de Jorge Quiroga (20012002). Ese mismo año, la población eligió de nuevo a Gonzalo Sánchez de Lozada, pero fue obligado a renunciar al año siguiente y fue substituido por el vicepresidente Carlos Mesa, el cual también abdicó de la presidencia en 2005. En su lugar asumió el presidente de la Corte Suprema de Justicia, Eduardo Rodríguez Veltzé, quien an-

2 La destitución de Fernando Collor de Mello (el primer presidente electo por el voto popular desde João Goulart en 1961) por el Congreso brasileño ocurrió en diciembre de 1992. 
ticipó para diciembre de ese mismo año las elecciones presidenciales, parlamentarias y de gobernadores. El vencedor en primera vuelta fue el candidato del Movimiento al Socialismo (MAS), Evo Morales Ayma, que fue posteriormente reelecto en 2009.

Ecuador es un país con una historia reciente de luchas políticas y de fuerte polarización ideológica que ha dado como resultado mandatos presidenciales cortos. Desde mediados de los años 1990, ningún presidente ha logrado concluir el mandato de cuatro años. El primer gobierno analizado es el de Sixto Durán Ballén (1992-1996), último presidente que terminó su mandato. Abdalá Bucaram Ortiz, presidente electo en 1996, permaneció únicamente 186 días en el cargo. Fue substituido por el presidente del Congreso Fabián Alarcón ${ }^{3}$, que anticipó unas elecciones presidenciales en las que resultó ganador Jamil Mahuad Witt (1998). Éste permaneció apenas un año y medio en el poder, destituido por un golpe militar con el apoyo de los indígenas. En su lugar asumió Gustavo Noboa, quien formó un gobierno de transición hasta la elección de Lucio Gutiérrez Borbúa (2002), el cual gobernó el país durante dos años cuatro meses hasta que el Congreso lo destituyó y lo obligó a huir del país. En mayo de 2005 asumió el cargo el vicepresidente Alfredo Palacio y administró el país hasta el 15 de enero de 2007, fecha en que Rafael Correa tomó posesión. En 2009 Correa convocó a nuevas elecciones presidenciales y fue reelecto con un amplio margen de votos.

De los veinticuatro presidentes electos, y considerando solo aquellos donde los ocurrieron elecciones regulares, es posible identificar seis caídas, lo que implica decir que aproximadamente $25 \%$ de los presidentes electos no lograron terminar sus mandatos. No obstante, al incluir a todos los que llegaron a ejercer la presidencia de sus respectivos países, incluso por sustitución de aquellos que no terminaron sus mandatos, el total de caídas presidenciales sube a diez, lo que equivale aproximadamente $41,66 \%$. La Tabla 1, presenta una lista resumida de las razones que propiciaron esas caídas.

Una maniobra del Congreso ecuatoriano impidió que la vicepresidente de Bucaram, Rosalía Arteaga, asumiera la presidencia. 
Tabla $N^{\circ} 1$

Motivos de las caídas presidenciales

\begin{tabular}{|l|l|l|l|}
\hline \multicolumn{1}{|c|}{ Año } & \multicolumn{1}{|c|}{ País } & \multicolumn{1}{c|}{ Presidente } & \multicolumn{1}{c|}{ Razón } \\
\hline 1992 & Brasil & Collor & Renuncia/Juicio Político \\
\hline 1997 & Ecuador & Bucaram & Destituido por incapacidad mental \\
\hline 1997 & Ecuador & Arteaga & Ignorada por el Congreso \\
\hline 2000 & Ecuador & Mahuad & Golpe \\
\hline 2001 & Argentina & De la Rúa & Renuncia \\
\hline 2001 & Argentina & Puerta & Renuncia \\
\hline 2001 & Argentina & Saá & Renuncia \\
\hline 2003 & Bolivia & Lozada & Renuncia \\
\hline 2005 & Bolivia & Mesa & Renuncia \\
\hline 2005 & Ecuador & Gutiérrez & Destituido por abandono del cargo \\
\hline
\end{tabular}

Fuente: Elaboración Propia

\section{SISTEMA POLÍTICO Y CONFLICTOS ENTRE PODERES}

En esta sección, se relacionan las principales discusiones teóricas acerca del sistema político de Argentina, Brasil, Bolivia y Ecuador y sus implicaciones para el surgimiento de crisis políticas e inestabilidad presidencial. La relación entre los tres poderes, Ejecutivo, Legislativo y Judicial, constituye uno de los principales elementos para la manutención de la gobernabilidad y, consecuentemente, de la estabilidad política y presidencial.

Amorim Neto (2006), al evaluar los sistemas presidencialistas de América Latina, observa una gran variedad institucional, como también lo muestran los trabajos de Shugart (1992), Sartori (1997), Jones (1995), Carey (2003) y Mainwaring (2003), entre otros, lo que -en interacción con la inclinación ideológica del presidente, el sistema partidario y las condiciones económicas- reflejaría una diversidad de patrones de gobernanza ${ }^{4}$. Estos, a su vez, podrían ser asociados al desempeño político y económico de los países de las Américas.

4 Santos, Almeida y Vilarouca (2008:24) definen la gobernanza como la capacidad de gobernar, o, en términos generales, como definir objetivos en primer lugar y enseguida tomar decisiones e implementar acciones para alcanzarlos. Los autores distinguen dos principales factores que afectan la gobernanza en la región: la distribución de los poderes legislativos entre el presidente y el Congreso y el grado de divergencia entre actores políticos detentores de poder de veto (especialmente entre el presidente y la tendencia mayoritaria en el Legislativo). 
De forma análoga, Anastasia, Ranulfo y Santos (2004:8) afirman que las instituciones: 1) afectan el comportamiento y los resultados políticos; 2) interactúan y producen efectos combinados sobre atributos deseables del orden democrático, tales como estabilidad política, rendición de cuentas y representatividad. Estos autores discuten el llamado dilema institucional de las recientes democracias latinoamericanas, que podría ser sintetizado en la dificultad de crear arreglos institucionales compatibles con sociedades que combinan heterogeneidades estructurales con pobreza y desigualdad social. Este dilema institucional sería traducido, de alguna forma, por la disyuntiva entre estabilidad y representatividad, o en la tensión de la ampliación del hiperpresidencialismo que podría generar mayor gobernabilidad en detrimento de la responsibidad/representación de los electores.

De acuerdo con Guillermo O’Donnell (1991), en el periodo inmediatamente posterior a la redemocratización de América Latina, en un contexto de severas crisis económicas y políticas, ocurrió en algunos países de la región una delegación explícita de poderes que resultó en una amplia libertad de acción de los presidentes democráticamente electos, lo que dio lugar al conocido concepto de "democracias delegativas". Para O’Donnell, la democracia delegativa ocurría en escenarios de baja institucionalización y representaba para los presidentes la ventaja de no tener prácticamente ninguna obligación de rendir cuentas, ni siquiera frente a otras instituciones fundamentales para la democracia como el poder Legislativo, Judicial, los partidos políticos y los movimientos sociales.

En muchos países, sin embargo, las consecuencias de ese nuevo modo de gobernar fueron perversas y proliferaron casos de la llamada estafa electoral ${ }^{5}$ que, por lo general, tuvo como implicaciones el aumento de la inestabilidad política, manifestaciones populares y caídas presidenciales. Es posible decir que una de las motivaciones de esa serie de reformas constitucionales en el sentido de retomar la representatividad de los ciudadanos sería una respuesta a los casos recientes del fenómeno.

Es posible identificar el conflicto entre poderes en la región, por ejemplo, en las sucesivas tentativas de reforma constitucional en los países en cuestión. Según Pachano (2004), las reformas constitucionales y legales implementadas en las dos últimas décadas acabaron fortaleciendo a los respectivos presidentes, llevando al Legislativo a

5 Stokes (2001), al analizar la implementación de las reformas neoliberales en la región, llama la atención sobre la ocurrencia de estafas electorales (policy switch), entendida como una acción estratégica de los representantes que ocultan sus intenciones reales y prometen la adopción de políticas atractivas para los electores durante las campañas y que, una vez electos, modifican radicalmente el sentido de sus políticas hacia orientaciones conservadoras y antipopulares. 
usar con mayor frecuencia el bloqueo de las iniciativas del Ejecutivo como estrategia de negociación política. Desde 1989, la principal reforma constitucional realizada en Argentina ocurrió en 1994; en ese mismo año hubo una reforma relevante en Bolivia, país que promulgó una nueva constitución por la vía del referéndum en 2009, mientras que en Ecuador se promulgaron constituciones en 1998 y 2008. En Brasil, la última constitución data de 1988, pero desde ese año han sido aprobadas 68 enmiendas que modificaron significativamente el contenido del texto constitucional original. Siendo así, el periodo reciente siempre ha ofrecido algún grado de incertidumbre para las prácticas políticas. La interferencia del Ejecutivo y del Legislativo, principalmente este último, en la formación y en la actuación del poder Judicial, por ejemplo, contribuyó de forma negativa para la estabilidad del país y para la resolución de conflictos. Pessanha (2002) afirma que los procesos de transición política que se realizaron en América Latina, en su mayoría, fueron consolidados con cambios constitucionales que, en algunos casos, sufrieron discontinuidades o profundas modificaciones a través de la introducción de sucesivas enmiendas y desobediencias al texto constitucional producido por mayorías parlamentarias sin la debida atención a los principios republicanos, responsables por los límites e instrumentos populares adecuados al control del poder político.

De acuerdo con Pachano (2006), una de las principales dificultades de los países de la región sería la formación de mayorías parlamentarias para la sustentación de gobiernos. En ese sentido, las negociaciones del Ejecutivo con el Legislativo, en especial con los partidos políticos, siempre constituyen un motivo de tensión y negociación política. Frecuentemente los partidos capitalizan las insatisfacciones populares para aumentar su poder de negociación, lo que eventualmente produce el inicio de una crisis o conflicto abierto entre poderes.

El argumento sería que, estando al frente del Ejecutivo, el partido tiene un margen de maniobra reducido, ya que para aprobar proyectos de ley y poner en práctica las políticas necesita del apoyo de la mayoría. Como generalmente no basta con la bancada oficialista en el Legislativo, el presidente necesita ceder en algunos puntos para avanzar en otros (Silva y Vieira, 2006:198), principalmente cuando está bajo presiones externas para la implementación de ajustes macroeconómicos. En esos casos el Ejecutivo necesita abandonar una "agenda preferida" para implementar una "agenda posible" y esa opción, la mayoría de las veces, promueve el desplazamiento del partido en el espectro ideológico.

Tales acontecimientos llaman la atención sobre la importancia del estudio de las coaliciones en el presidencialismo como sustento de los mandatarios. La literatura es 
prolífica en discutir la estabilidad política y su relación con las mayorías parlamentarias y los sistemas de partidos y sus autores están divididos en dos lados opuestos: los que creen que gobiernos con apoyo minoritario en el Congreso llevan necesariamente a situaciones perjudiciales para la gobernabilidad -Mainwaring (1993) y Linz (1994), entre otros- y los que creen que gobiernos minoritarios no necesariamente son la causa de una merma en la gobernabilidad -Chasqueti (2001), Cheibub (2002), Negretto (2003) y Jiménez (2007).

El argumento del primer grupo de autores, de visión más pesimista, sería que los presidentes no tendrían incentivos para formar coaliciones y que, aunque llegaran a formarse, estarían compuestas por partidos indisciplinados. Otra afirmación común sería que presidentes minoritarios serían legislativamente ineficaces, generando muchas veces impases sin solución constitucional. Finalmente, cuando ninguna coalición se forma no existe alternativa al impase legislativo, ni a la parálisis y, en último caso, a la quiebra del sistema democrático.

Por su parte, el segundo grupo, más optimista, trata de demostrar que la inestabilidad política en el presidencialismo no es una condición intrínseca al sistema, sino que surge sólo en arreglos institucionales específicos, como en el caso de presidentes minoritarios que no logran o no quieren formar coaliciones de gobierno en sistemas multipartidarios. Según los autores, con los presidentes que son capaces de formar coaliciones mayoritarias la tasa de fracaso es relativamente baja y aceptable.

De acuerdo con Badillo (2007:156), los mecanismos para gobernar con estabilidad política están directamente relacionados con la capacidad de ambos poderes (Legislativo y Ejecutivo) para formar coaliciones partidarias y sancionar las iniciativas de ley que son parte de la agenda del gobierno. La autora subraya que la institucionalización del sistema partidario es uno de los factores importantes para la formación de coaliciones legislativas. Los sistemas partidarios poco institucionalizados -como es el caso de Ecuador- tendrían poca disciplina y baja cohesión interna para formar coaliciones de mayor duración. Jiménez Badillo (2007) utiliza la definición de Mainwaring y Scully (1997) para determinar cuándo un sistema de partidos es institucionalizado: 1) hay competencia regular entre partidos;2) los partidos poseen raíces estables en la sociedad; 3 ) los partidos y las elecciones son los mecanismos que determinan quién gobierna; 4) las organizaciones partidarias son relativamente sólidas. Argumenta Jiménez Badillo (2007), que al analizar los sistemas presidencialistas latinoamericanos, sustenta como su principal argumento que mientras mayor sea la distancia ideológica entre los partidos y el presidente, menores serán las posibilidades de que se formen coaliciones legislativas. De esa manera se acerca al argumento de 
Chasquetti (2001) y Cheibub (2002) sobre la importancia de la polarización del sistema político como uno de los principales factores para la inestabilidad de los regímenes presidencialistas.

Por lo tanto, la lógica de la negociación entre el Ejecutivo y el Legislativo sería fundamental para determinar las crisis políticas. Cheibub, Przeworski y Saiegh (2004) afirman que un presidente puede gestionar un gobierno minoritario sin crisis en determinadas situaciones siempre que logre formalizar coaliciones mayoritarias y estables. En caso de que no llegue a ese objetivo, puede hacer cambios en su gabinete y formar una nueva coalición. Mientras mayor sea el poder de agenda y la capacidad de imprimir restricciones a la forma de votar del legislativo, mayor sería la capacidad de controlar el resultado.

Negretto (2003:78) también se enfrenta al argumento en que parte de la literatura afirma que los presidentes minoritarios en el Congreso no son estables. Según ese autor, deberían ser considerados tres factores para analizar el desempeño del presidente: 1) el tipo de políticas públicas que propone el partido gobernante; 2) la capacidad presidencial para vetar medidas aprobadas por el Congreso, y 3) el estatus legislativo de los partidos incluidos en el gabinete. El autor afirma que el potencial de conflicto en un régimen presidencialista aumenta cuando el partido del presidente pierde el apoyo tanto del legislador medio como del legislador que definirá la situación de veto y, simultáneamente, no se logra la formación de un gabinete de coalición que sustente una mayoría legislativa a favor del partido del presidente.

En ese sentido, el análisis de las fuerzas políticas distantes del centro se muestra importante, pues tal configuración constituye un factor relevante de inestabilidad. Creo en la posibilidad de utilizar el concepto de Sartori (Santos, 1986:26) denominado escala de promesas. Los partidos que entran al juego de las promesas irreales no están respetando las reglas usuales de la competencia y, consecuentemente, el juego político es conducido por una escalada incesante del conflicto. Tales partidos (o fuerzas políticas) tienen pocas esperanzas de ocupar el gobierno y por esa razón no respetan los límites en sus promesas con la intención de desestabilizar al centro.

No obstante, creo que la presencia de la polarización política a niveles elevados no necesariamente conduce a crisis políticas, una vez que la literatura ya demostró que la posibilidad de formar coaliciones amplias para sustentar la gobernabilidad del Ejecutivo forma parte de la dinámica del presidencialismo. 
Además de la conocida polarización ideológica, la polarización ocurre en los países analizados también a través de la dinámica de participación o no en el gobierno. Esa oposición habría sido recreada y fortalecida en gran parte por la acción popular en las protestas y revueltas que ocurrieron por la vía de los piqueteros, los indígenas y los cocaleros, con la acción de un gran contingente de ciudadanos contrarios al ajuste estructural y a los partidos que implementaron las reformas. La participación popular en las manifestaciones y destitución de presidentes, el surgimiento de nuevos partidos políticos y de nuevos líderes habrían sido el principal motor del cambio. El principal tema de la polarización, en ese momento, pasó a ser entre los que defendían y/o implementaron las reformas estructurales neoliberales y los que estaban en contra.

Otro argumento que puede ser usado cuando reflexionamos sobre la inestabilidad política consiste en la presencia de una relevante polarización geográfica en alguno de los países analizados. Las elecciones presidenciales en Ecuador en 1998 retratan bien esa cuestión. En aquella ocasión, una de las principales armas de la campaña de los dos candidatos principales era exactamente el fortalecimiento de sus bases. Según datos del Tribunal Supremo Electoral ecuatoriano, los resultados de la segunda vuelta demostraron bien esa diferencia: en la región serrana, Jamil Mahuad logró 66\% de los votos y Gustavo Noboa 33\%; en la zona costera ocurrió el fenómeno contrario, ya que Noboa obtuvo $62,7 \%$ contra $37 \%$ de Mahuad. En la provincia costera de Guayas, Mahuad creció solamente 2,05\% entre la primera y la segunda vuelta. De forma análoga, los conflictos entre la llamada "región de la media luna" y los demás departamentos bolivianos, o incluso entre la posición conservadora y el gobierno central de Morales ponen en evidencia la importancia de la polarización geográfica en algunos países de la región. A lo largo de 2008 esa polarización casi provoca una guerra civil en el país. En el caso argentino la dinámica de la polarización geográfica está en la distinción entre la capital, Buenos Aires, y el resto del país. En el caso brasileño, sin embargo, no encontramos evidencias relevantes de polarización geográfica y ninguna indicación de la existencia de algún tipo de inestabilidad política motivada por tal particularidad.

Uno de los señalamientos de la teoría que discute la polarización política es el concepto de parálisis decisoria, la cual se produce por la existencia de una fuerte polarización entre los actores en alguno de los países que serán analizados. El modelo (analítico formal de competencia política) establece, mediante la descripción de sistemas polarizados, que una crisis de parálisis decisoria se vuelve el resultado más probable de la confrontación política cuando los recursos de poder se dispersan 
entre los actores radicalizados en sus posiciones, de tal forma que se establece lo que denomino equilibrio político (Santos, 2006:10).

Al explicar el fenómeno de la parálisis decisoria, Santos sugiere que los procesos que la produjeron fueron los siguientes: 1) la fragmentación de los recursos de poder acompañada de la radicalización ideológica; 2) la fragilidad y la inconstancia de las coaliciones que se formaron en el parlamento, 3) la inestabilidad gubernamental, definida como la rotación constante de ministros y titulares de agencias estatales. El referido autor cree que los procesos políticos pueden efectivamente ser considerados variables independientes y atrae la atención hacia el examen de las tendencias empíricas que no se identifican de otra forma. No serían, por lo tanto, únicamente subproductos de tendencias macrosociales o macroeconómicas. La naturaleza de los resultados del conflicto depende de la adinámica de la competencia institucional, que será responsable, en última instancia, por la mayor o menor capacidad del sistema político como un todo para organizar las acciones colectivas destinadas a solucionar problemas.

El concepto de parálisis decisoria es semejante al de deadlock. De acuerdo con Cheibub (2002), la ocurrencia de situaciones de deadlock dependería de una combinación de factores políticos e institucionales. Por un lado, dependería de la distribución de curules en el Congreso o, más específicamente, de la distribución de las curules dominados por el gobierno. Por otro lado, dependería de los requisitos institucionales necesarios para el veto presidencial. Tales condiciones serían: la existencia de poder de veto presidencial en la legislación, el tipo de mayoría -simple o calificada- necesaria en el Congreso para anular el veto presidencial y, finalmente, si son necesarias votaciones en una o dos cámaras. El deadlock no ocurriría cuando el control de la mayor parte de las curules legislativas pertenece al presidente.

Uno de los ejemplos paradigmáticos de parálisis decisoria -o deadlock- puede ser percibido en Ecuador, en el breve mandato de Lucio Gutiérrez. El excoronel fue electo con un discurso reformista, contrario a los ajustes macroeconómicos y a la dependencia externa, contando con el apoyo de una coalición de centro-izquierda que incluía al partido indígena Movimiento Pachakutik (PK). Sin embargo, ya en los primeros meses el gobierno tomó una dirección opuesta a lo prometido. Ese movimiento acabó generando la pérdida gradual de su base parlamentaria, de manera que la bancada oficialista en el Congreso -que es unicameral- se redujo a solamente ocho diputados de un total de cien. Enseguida ocurrió un progresivo desplazamiento ideológico de las relaciones de fuerza partidaria y la recomposición de la base parlamentaria necesaria para una mayor estabilidad y la consecución de 
políticas de ajuste fiscal. En ese sentido, Gutiérrez incorporó siete nuevos ministros de los partidos de centro derecha, recuperando la mayoría en el Congreso.

Meses después, los partidos que apoyaron a Gutiérrez en el inicio de su mandato fueron los mismos que promovieron una ofensiva de oposición, iniciando un juicio político al presidente. A partir de marzo de 2005, el potencial de conflicto entre las fuerzas políticas ecuatorianas alcanzó un nivel en que los costos de la supresión del adversario se redujeron frente a los costos de la tolerancia, rompiéndose así una situación de "seguridad mutua" ${ }^{6}$. Con el apoyo de la nueva mayoría, Gutiérrez implementó una serie de reformas, entre ellas modificaciones en las Cortes Supremas del país, que son partidizadas, retirando a los jueces afiliados a los partidos de oposición y sustituyéndolos por magistrados de partidos fieles al régimen. Enseguida, líderes de la oposición convocaron a los ecuatorianos a las calles pidiendo la renuncia inmediata del presidente. Finalmente, los estudiantes invadieron el edificio del Congreso Nacional, mientras los diputados destituían al entonces presidente del Congreso, seguido de la votación de la destitución de Gutiérrez bajo la alegación de abandono del cargo, a pesar de que el entonces presidente estuvo todo el tiempo en su oficina. En el contexto regional, la intervención en la Suprema Corte es uno de los recursos más utilizados por los gobernantes que desean un amplio control del país revestido de una supuesta apariencia de constitucionalidad. Del mismo modo actuó Menem, aumentando el número de jueces de la Suprema Corte Argentina y estableciendo un amplio control del país para realizar un paquete de reformas neoliberales.

Sin embargo, uno de los aspectos que es necesario destacar es que para que las intervenciones del Ejecutivo en el poder Judicial puedan tener éxito es necesario contar con una coalición oficialista necesariamente mayoritaria en el Legislativo. Fue eso lo que pasó en el caso argentino, una vez que el aumento del contingente de jueces de la Suprema Corte Argentina ocurrió en un momento extremadamente favorable a Menem, que contaba con un amplio apoyo legislativo para sus medidas, además de apoyo popular. Como quedó demostrado, lo mismo no ocurrió en el caso de Gutiérrez en Ecuador, ya que tuvo que enfrentar una fracción significativa de legisladores de la oposición que se oponían a sus medidas así como sucesivas manifestaciones por parte de los movimientos sociales, volviendo insustentable su permanencia en el poder.

Dahl (2005: 37) afirma que entre más bajos sean los costos de la tolerancia, mayor será la seguridad del gobierno y que mientras más altos sean los costos de su supresión, mayor será la seguridad de la oposición. 
En el caso brasileño, a excepción de Fernando Collor de Mello, los últimos presidentes siempre han logrado formar coaliciones mayoritarias que les permitieron las condiciones mínimas de gobernabilidad.

Conforme fue explicado, los intentos de intervención en el poder Judicial en los países analizados refuerzan un aspecto propio del funcionamiento de las Cortes Supremas en las democracias en cuestión: el hecho de que muchas veces no actúan como fiscales de la ley o para el control de la constitucionalidad, sino efectivamente como otra instancia de negociación. Tal hecho sería un grave problema para la teoría democrática tradicional y para la estabilidad política de los países analizados.

No obstante, después de ese momento de polarización extrema y tomando como base la sugerencia teórica de la literatura estadounidense, tal vez podamos afirmar que lo que ocurrió desde entonces fue un periodo de reacomodo del conflicto en términos del teorema de Downs (1999), con partidos de nuevo en proceso de realineamiento hacia el centro político. Ese movimiento se habría consolidado a partir de la mitad de los años 2000, pero de formas diferentes en la región. Mientras en algunos países, como Brasil, ya no se identifica una gran diferencia ideológica entre gobierno y oposición, en otros como en Bolivia y Ecuador, esa distinción persiste, pero aún en diferentes grados.

Por lo tanto, la manera mediante la cual el sistema político está estructurado en cada país de la región será el factor determinante para la definición de su estabilidad. Factores como la importancia de los poderes de veto y decreto de los presidentes y la polarización de los sistemas partidarios, además del análisis de la distancia entre la posición del presidente y la mayoría parlamentaria también serían determinantes para la estabilidad política y para la estrategia de formación de los gabinetes.

\section{MOVIMIENTOS SOCIALES}

En esta sección se discute el papel de los movimientos sociales para la definición tanto de la estabilidad política como de la estabilidad presidencial en Argentina, Brasil, Bolivia y Ecuador. Estructuro la discusión considerando dos argumentos principales: 1) la actuación de los movimientos sociales en la promoción de las principales movilizaciones contra presidentes, y 2) la organización de partidos políticos que funcionan como brazos políticos para la representación institucional de sus intereses. 
Melucci (2001) afirma que un movimiento social no es la respuesta a una crisis, sino la expresión de un conflicto, manifestado en su organización, lo que implicaría una ruptura de los límites de compatibilidad del sistema al cual la acción se refiere. En este universo están inscritos tanto la actuación de los movimientos sociales de linaje clásico, como por ejemplo el movimiento sindical, y también los llamados nuevos movimientos sociales (NMS), como los movimientos étnicos y piqueteros.

De acuerdo con autores como Mirza (2006) y Silva (2011), la acción de los movimientos sociales latinoamericanos podría ser resumida en una idea central: con el fin del periodo autoritario y el retorno de la democracia, se crearon nuevas expectativas en los ciudadanos, quienes después de convivir con la represión de las dictaduras en casi toda la región, aspiraban a ejercer derechos inherentes a una democracia libre, acompañado de la mejoría en las condiciones de vida después de décadas de pobreza y desigualdad social. Sin embargo, la clase política logró responder a esas demandas de la forma esperada. Como resultado, los ciudadanos comenzaron a dejar de creer en el modelo de representación tradicional de los partidos políticos y, por extensión, en la democracia como sistema: así, se manifestaron en sucesivos levantamientos los indígenas; en piquetes y marchas, los desempleados; en ocupaciones de tierra y movilizaciones, los sin tierra; en huelgas, bloqueo de vías y manifestaciones en las calles, los trabajadores y campesinos (Mirza, 2006: 13).

Argumentando en la misma dirección de Mirza (2006), pero ampliando la discusión hacia el concepto de estabilidad presidencial, Hochstetler (2007) afirma que la presencia o ausencia de protestas populares en las calles constituye un fenómeno esencial para la salida de los presidentes en Sudamérica. Según la autora: todas las movilizaciones exitosas de caídas presidenciales incluyeron participaciones de la sociedad civil que exigían en las calles la salida de los presidentes (Hochstetler, 2007: 16). Aun así, la participación popular en movilizaciones no constituye el único medio de acción ni tiene efecto obligatorio, ya que en diversos momentos la ocurrencia de grandes movilizaciones no necesariamente provocó la destitución de presidentes. Sin embargo, al parecer, todas las caídas presidenciales que tuvieron como motor principal la acción del Legislativo contaron también con una fuerte actuación de los movimientos sociales que organizaron las protestas de la sociedad civil. Es posible afirmar, por lo tanto, que la participación de la sociedad civil y los movimientos sociales puede ser considerada una condición necesaria, pero no suficiente, para las caídas presidenciales en el periodo analizado.

Siendo así, se define la actuación de los movimientos sociales en relación con la estabilidad presidencial en el periodo posterior a 1989 en tres tipos: 1) para destituir 
presidentes; 2) contra el Estado y el sistema político en general (expresado en el lema “que se vayan todos") y 3) por la manutención de presidentes (oposición al golpe contra Chávez en 2002). De acuerdo con Pérez Liñán (2008), los militares eran en el pasado el principal factor de fuerza capaz de imponer la salida de un presidente. Ahora bien, el retiro del ejército del campo político tuvo dos consecuencias fundamentales: 1) los movimientos sociales se transformaron en el factor fundamental capaz de desestabilizar un gobierno, a través de formas de protesta confrontacional o violenta; 2) la capacidad represiva del Estado fue limitada por la creciente resistencia del ejército en ejercer funciones parapoliciales. Esta situación inició una escena de poder popular que, combinada con el marco de las instituciones democráticas, no tiene precedentes en América Latina.

El "pueblo", por lo tanto, conquistó así un papel preponderante en la explicación de las crisis presidenciales, no como referencia a una masa amorfa y manipulada, sino como una categoría que representa la ciudadanía movilizada, no como la representación de una turba anómica, sino como la manifestación política de los movimientos sociales. Identificando la importancia de las protestas sociales en el nuevo contexto histórico, Zamosc (2004) denominó los casos de inestabilidad presidencial como "juicios políticos populares”. Tales acontecimientos generaron una dinámica compleja entre la sociedad civil, los sectores movilizados y los actores institucionales. Como regla general, en momentos de crisis política mayor, esos movimientos de protesta buscan la destitución del presidente en ejercicio, pero no poseen un plan coordinado para substituirlo por otra figura. Con el objetivo de ejercer de alguna forma un mecanismo de rendición de cuentas, las protestas se enfocan contra el gobierno, pero no contra el régimen democrático. Por ese motivo, autores como Zamosc (2004), Llanos y Marsteintredet (2010) y Samuels y Hochstetler (2011) concluyen que cuando percibimos la movilización popular simplemente como una disrupción del orden legal, corremos un riesgo serio de ver la democracia solamente en sus aspectos formales, ignorando la dimensión sustantiva.

Para Mirza (2006), la mayoría de los partidos políticos tradicionales demostró la incapacidad para asumir la conducción eficaz del proceso de reconstrucción del Estado con el fin del periodo autoritario. Por lo tanto, los nuevos movimientos sociales latinoamericanos configuraron nuevos ejes del conflicto social. Por eso, se necesita un estudio cuidadoso de sus estrategias y formas de lucha, de sus demandas e interpelaciones, de sus victorias y conquistas sociales. En los años 80, los movimientos sociales comenzaron a ocupar el espacio de los partidos, irrumpiendo en el ambiente de la política institucional. Los espacios, por lo tanto, se confunden - una 
de las razones para la necesidad de estudiar las relaciones entre sistema político y movimientos sociales.

Este tipo de desarrollo teórico percibe a los nuevos movimientos sociales como fundamentales para la reconstrucción de un modelo democrático incluyente que vaya más allá de la democracia representativa tal y como se lleva a la práctica actualmente. De acuerdo con Coutinho, Mireles y Delgado (2008), la movilización colectiva que se observa en Sudamérica en este inicio de siglo ocurre principalmente contra la exclusión, sea o no en relación directa con el proceso neoliberal. En ese sentido, los movimientos sociales de la región estarían luchando por alzar su voz, por la inclusión social y el reconocimiento de sus derechos, saliendo así de los márgenes. Al mismo tiempo, el actor colectivo sudamericano estaría luchando también para ser parte de procesos decisorios, muchos de ellos cruciales para determinar el rumbo nacional y con repercusión en los ámbitos regional, internacional y hasta global. Los anhelos de Dahl en "Poliarquía" (1997), sobre inclusión, están vivamente presentes en ese argumento. Mirza (2006) afirma que partidos políticos, movimientos sociales y todos los actores sociales están estrechamente vinculados con la construcción y manutención del delicado equilibrio democrático. La repartición del poder en una democracia representativa no es un acto único que se ejecuta al día siguiente de las elecciones, sino el producto de una interacción permanente cuya correlación de fuerzas depende de factores económicos, políticos y socioculturales (para más información ver: Pérez Flores; Cunha Filho y Coelho 2010).

Las variaciones de esa correlación de fuerzas serán distribuidas según la posición de control y manejo de recursos estratégicos -económicos, institucionales, simbólicos, políticos-, por la capacidad de articulación entre los actores y sus vehículos de comunicación interna y externa, por el apoyo de los ciudadanos y por el grado de legitimidad acumulado. Las alianzas de clase y sectores sociales, en ese sentido, se manifiestan en las articulaciones entre los movimientos sociales y partidos políticos.

En los casos de Bolivia y Ecuador, el énfasis recae sobre los movimientos étnicos. Inicialmente, durante el siglo XX los indígenas, cuando fueron incorporados al Estado, siempre lo fueron en calidad de campesinos y vinculados a una concepción de lucha de clases. A partir de finales de la década de 1970, se reconsideraron tales movimientos y la identidad étnica comenzó a ganar fuerza (Van Cott, 2005).

En el caso boliviano, los movimientos indígenas siempre han estado ligados al movimiento sindical y las demandas de carácter étnico fueron agregadas posteriormente. Aun así, no es posible decir que hay un movimiento indígena cohesionado, ya que 
hay una clara separación entre los propios grupos indígenas, específicamente los movimientos indígenas Katari y los indianistas (Camargo, 2007).

También el caso ecuatoriano está ligado en su génesis a los movimientos campesinos y a las federaciones sindicales. Pero desde la fundación de la Confederación de las Nacionalidades Indígenas Ecuatorianas (Conaie) en 1979, el debate adoptó la tonalidad de las demandas étnicas (Yashar, 2005). En 1990, esta organización asume el protagonismo con los diversos bloqueos que paralizaron el país y que desde entonces han ocurrido con bastante frecuencia. ${ }^{7}$ En 1996 fue creado el movimiento Pachakutik (PK), brazo político de la Conaie y desde entonces la principal estrategia electoral utilizada por el Pachakutik fue el apoyo a otros candidatos en las elecciones presidenciales y no la constitución de una candidatura propia. ${ }^{8}$

De esa manera, los movimientos sociales de carácter étnico en Ecuador y Bolivia se distinguen de los movimientos sociales tradicionales del pasado por nuevas reivindicaciones y su formación más amplia (Dávalos, 2005). Están formados en su mayoría por sectores excluidos de la población y se han transformado, a lo largo de los años, en verdaderas potencias sociales en la arena política de sus respectivos países. Están focalizados en las nuevas demandas impuestas por los efectos de la globalización y por mayor inclusión social y política. Después de años de experiencia democrática, estos movimientos han ganado peso, entraron al juego político formal y han llegado a ocupar la Presidencia, como en el caso boliviano.

En ese contexto se destaca el surgimiento de nuevos partidos en el escenario político de la región, principalmente de los que han sido fundados a partir de movimientos sociales o de fuerzas de la sociedad civil. En países como Bolivia y Ecuador, por ejemplo, por primera vez en la historia ha sido posible la constitución de partidos políticos indígenas con efectiva fuerza legislativa y poder de negociación.

Según Jatobá y Epsteyn (2007: 55) en el caso argentino, los diferentes movimientos piqueteros, grupos heterogéneos de desempleados surgidos en el contexto del activismo anterior a la crisis del 2001, podrían ser considerados como los grupos políticos más organizados, combativos y contestatarios del país. De manera semejante a los movimientos sociales andinos, sus movilizaciones masivas incluirían en

Ecuador se destaca en la región como uno de los pocos países que han conseguido articular una confederación indígena unificada que moviliza una identidad étnica y programática, aunque eso no haya significado, todavía, una representación parlamentaria proporcional o la elección de un presidente indígena, como ya ocurrió en Bolivia.

8 La primera candidatura a la presidencia lanzada por Pachakutik fue la de Luis Macas en 2006. 
muchos casos el bloqueo de puentes y carreteras de crucial importancia estratégica para hacer valer sus demandas frente al gobierno. Tales protestas fueron acompañadas por lemas que expresaban total repudio a la clase política tradicional, anunciados por el slogan que se vayan todos. Aun de acuerdo con esos autores, otro fenómeno inédito surgió de la crisis económica y de la agitación política y social de Argentina en el inicio de los años 2000: la ocupación de fábricas por parte de empleados despedidos.

De acuerdo con Coletti (2004), el Movimiento de los Trabajadores sin Tierra (MST) fue el movimiento social popular que más creció en Brasil en la década de 1990 y se volvió el principal foco de resistencia a la política neoliberal implementada en el país desde la elección de Collor (1989). El autor sostiene que el MST creció en una coyuntura de reflujo para la mayoría de los otros movimientos sociales populares del país, en especial para el movimiento sindical combativo articulado en torno de la Central Única de Trabajadores (CUT), considerada por una vasta literatura sobre el tema como un caso paradigmático de construcción del poder sindical en América Latina.

Nacido oficialmente en 1984, el MST ha luchado por la reforma agraria y por la justicia social en un país que posee uno de los mayores índices de concentración de tierras y riqueza del planeta. Sus principales instrumentos de lucha son las ocupaciones de tierra, las acampadas, las marchas, las ocupaciones de edificios públicos, los saqueos y las manifestaciones públicas, utilizados como instrumentos de presión sobre el gobierno para la desapropiación de tierras y asistencia técnica financiera de los asentados. Sin embargo, el MST no posee una ideología de confrontación directa contra el Estado y no ha participado de manera decisiva en movilizaciones contra presidentes. No obstante, desde la elección de Lula en 2002, el MST ha perdido parte considerable de su autonomía, alineándose progresivamente al Estado y al Partido de los Trabajadores (PT). De acuerdo con Fernandes (2006:8), los movimientos campesinos han participado en los nombramientos para diversos cargos de segunda importancia en el gobierno, lo que pone en evidencia su estrecha relación con el Ejecutivo.

En ese periodo de seguidas crisis económicas y aumento de la polarización, es posible destacar también el aumento de las crisis políticas y la caída de varios presidentes, con amplia participación de la sociedad civil y de los movimientos sociales. Innúmeras fueron las movilizaciones, protestas y huelgas emprendidas. Los movimientos sociales organizados, con el apoyo de la población y de determinados partidos políticos, llegaron a cuestionar frontalmente el orden institucional de sus respectivos países, como en el caso de la invasión de ciudadanos ecuatorianos al Congreso Nacional y al Palacio Presidencial de Carondelet, que terminaron siendo acciones decisivas para la caída tanto de Jamil Mahuad (2000) como de Lucio Gu- 
tiérrez (2005). También en los episodios bolivianos las protestas populares llegaron a sitiar la capital, La Paz, lo que arrojó un saldo muy grande de muertos y heridos. El principal motivo de esas acciones era la renuncia consecutiva de dos presidentes: Gonzalo Sánchez de Lozada (2003) y Carlos Mesa (2005). En relación con Argentina, en especial, la actuación popular de los piqueteros y de los desocupados fue fundamental para la profundización de la crisis política que resultó en sucesivas caídas de presidentes entre 2001 y 2003.

Aquí, por lo tanto, cabe una reflexión entre la línea de acción de los partidos políticos tradicionales y de los movimientos políticos. Uno de los señalamientos de la vasta literatura sobre partidos políticos sería que estos tienen un mayor compromiso con el juego y las instituciones democráticas, lo que no sería compartido en la misma medida por los movimientos sociales. Mainwaring y Scully (2008), por ejemplo, argumentan que los sistemas partidarios institucionalizados facilitan una gobernanza democrática efectiva. Para los autores, un sistema partidario institucionalizado provee estructura para la democracia política en la arena electoral y en la legislatura. De ese modo, si los mismos partidos participan en la contienda electoral a lo largo de un periodo considerable de tiempo terminan dándole estabilidad al sistema, generando más previsibilidad en la acción de los actores y en las decisiones políticas, ya que los partidos ayudan a reducir los costos de información y transacción.

Resalto, sin embargo, que cuando los movimientos sociales no llegan al poder, se convierten en influyentes grupos de presión que no siempre se insertan en la lógica democrática, llegando a la utilización de diversas tácticas que podrían ser consideradas más radicales, como forma de negociación política. ${ }^{9}$ De acuerdo con Segovia (2004), la inestabilidad económica y política de esos países desde el regreso de la democracia promovió la consolidación de espacios extrainstitucionales donde los movimientos sociales discuten, rechazan y presentan alternativas a la aplicación de las reformas económicas y políticas del Ejecutivo. De este modo, los movimientos sociales habrían conquistado y consolidado sus propios canales de participación, ya sea aprovechando los espacios institucionales o impulsando nuevas vías de contestación extrainstitucional, como ocurrió, por ejemplo, en la toma de carreteras, bloqueo de vías, cacerolazos e invasiones al parlamento. Lo que percibimos en esos

9 En el caso ecuatoriano, la Conaie/Pachakutik actúa en dos frentes. Como partido, influenciando directamente en la dinámica institucional: proponiendo leyes, promoviendo vetos a las iniciativas del Ejecutivo, iniciando el proceso de juicio político contra el presidente; y como movimiento social, siendo el principal promotor de las huelgas y manifestaciones que paralizaron al país, con su alto poder de negociación. 
países fue, por lo tanto, la articulación del discurso histórico, sindical y étnico cultural con los contenidos antineoliberales en las décadas de 1990 y 2000.

Tales movimientos sociales se constituyeron como verdaderos grupos de presión organizados en torno de sus intereses. La mayor prueba de la relevancia de su actuación consiste en la cristalización de sus principales demandas en intereses nacionales, integrándose en buena medida a la agenda política de sus países, como por ejemplo, en la instauración de las Asambleas Constituyentes en Bolivia y Ecuador ${ }^{10}$; en las protestas contra la profundización de las relaciones con Estados Unidos y la firma de tratados de libre comercio. Percibimos que, en el caso argentino, las medidas ortodoxas del Ejecutivo en la economía fueron blanco de protestas que terminaron por modificar la orientación de la agenda política de los mandatarios, como ocurrió con el gobierno de Kirchner (2003-2008), quien integró al gobierno a algunos de los miembros destacados del movimiento de los piqueteros y de desocupados, con el objetivo declarado de insertar a la sociedad civil a la discusión política y a la planeación del país. Sin embargo, la adhesión y participación de líderes piqueteros y desocupados en el gobierno de Kirchner también ha sido considerada por muchos analistas como una especie de cooptación de esos liderazgos con el claro objetivo de evitar que su actividad política se vuelque contra la política del nuevo presidente en momentos de crisis. Esa estrategia habría causado la desmovilización de ambos movimientos, los cuales se habrían disciplinado de cierta manera ante el gobierno a cambio de cargos y de la concesión de diversos beneficios (Amorim y Arias, 2009: 247).

No obstante, al discutir específicamente el ambiente político argentino, Wolff (2007) afirma que contemporáneamente la influencia de los piqueteros habría quedado confinada a la participación individual de sus líderes en el parlamento o en gabinetes del Ejecutivo. Por un lado, eso sería debido a la capacidad del partido peronista para adaptarse a los cambios circunstanciales y absorber a las fuerzas sociales y, por otro, el hecho de que la categoría social de los "trabajadores desempleados" no representaría un clivaje social viable para la construcción de una organización política diferenciada. Con todo, el autor argumenta que no habría dudas de que esos países hoy en día están más abiertos a la participación de esos sectores populares que en el pasado.

De ese modo, la gran importancia de los movimientos sociales y de la sociedad civil en los procesos de crisis política y principalmente en la definición de estabilidad presidencial demuestra la necesidad de la reflexión sobre el papel real de ese contingente en los países analizados y, en una esfera mayor, en el presidencialismo.

10 Para mayor información ver: Pérez Flores, Cunha Filho, Coelho, 2010. 
Al mismo tiempo que el Ejecutivo utiliza estrategias en las que se disminuye o incluso se ignora prácticamente la representatividad popular situada en el Legislativo en nombre de la gobernabilidad, los representados aparecen como fuerza política definidora de la manutención de los presidentes.

De esa manera, lejos de ser considerados como elementos desestabilizadores de la democracia en la región, los movimientos sociales pueden ser calificados en gran parte como los principales defensores de la soberanía popular, utilizando las movilizaciones contra presidentes como una forma de restablecer el equilibrio político de sus países.

\section{CONCLUSIÓN}

La propuesta de este artículo fue discutir cómo la conformación de las instituciones políticas y la actuación de los movimientos sociales y de la sociedad civil influyeron directamente en el advenimiento de crisis políticas y en la caída de presidentes en los últimos 20 años en Argentina, Brasil, Bolivia y Ecuador. Para ello, se realizó una revisión crítica de la literatura contemporánea, indicando los diversos enfoques existentes y sus puntos de encuentro y divergencia.

En general, sobre el desempeño del sistema político en los países analizados, Anastasia, Ranulfo y Santos (2004:9), que afirman que los efectos combinados de las instituciones políticas pueden producir una distribución de los poderes de agenda y de veto entre los actores que expresa un punto de equilibrio entre estabilidad, rendición de cuentas y representatividad, o sea, el llamado punto de equilibrio de la democracia. Ese punto de equilibrio sería afectado por la variación de las condiciones sobre las cuales las instituciones políticas deben operar. Según los autores, existen evidencias de que determinadas combinaciones entre instituciones y condiciones serían capaces de producir tal punto de equilibrio.

En el mismo sentido, Hagopian y Mainwaring (2005) subrayan que la vulnerabilidad de un régimen político estaría directamente relacionada a la creación de instituciones que regulen el conflicto distributivo de los países de América Latina: en otras palabras, regímenes democráticos son vulnerables especialmente cuando fallan en resolver las presiones oriundas de las necesidades de ciudadanos y no desarrollan mecanismos de inclusión y representación para compensar las ineficiencias en sus desempeños. Hofmesteir (2004) dirige su argumento en el mismo sentido cuando afirma que la inestabilidad generada por la alternancia entre periodos democráticos y golpistas en el pasado reciente en prácticamente todos los países de América Latina representa una 
definitiva dificultad para legitimar medios sólidos de gobernabilidad. Esos procesos, según el autor, se desarrollan en un contexto de enorme precariedad institucional que resulta en el desvío del funcionamiento de los respectivos sistemas políticos que no lograron crear mecanismos sólidos de acceso a derechos civiles, sociales y políticos.

Como quedó demostrado a lo largo del texto, en algunos momentos las instituciones democráticas no fueron capaces de dirimir o regular los conflictos en escenarios de crisis, lo que muchas veces provocó un conflicto abierto entre los tres poderes o incluso entre el presidente y parte considerable de la sociedad civil, principalmente por la vía de los movimientos sociales. Así, las sucesivas manifestaciones, levantamientos, huelgas, entre otras formas de movilización, han servido en realidad como una especie de rendición de cuentas popular en la búsqueda de una mayor correspondencia entre los deseos de los ciudadanos y la efectiva actuación de sus representantes. Si en algunas ocasiones fue posible retomar el control del arreglo distributivo de las fuerzas políticas y alcanzar una solución a las crisis, en otras las demandas eran tantas y tan intensas que el único camino posible consistía en la destitución de presidentes democráticamente electos. En ese sentido, es interesante el análisis del caso brasileño, que, en el mismo periodo, y a pesar de innúmeras crisis políticas y económicas, logró manejar mejor sus conflictos.

Independientemente del tipo de crisis, Negretto (2003) afirma que parece claro en la mayoría de los casos de caída de presidentes que tanto el Congreso como los mandatarios percibían la eliminación del otro como la solución para la crisis. Bajo esa perspectiva, la renuncia forzada o incluso el juicio político a los presidentes, por un lado, y la sustitución o disolución del Congreso por los mandatarios, por el otro, pueden ser percibidas como resultados simétricos no cooperativos. De acuerdo con Wolf (2007), el llamado “juicio político popular”, o sea, la deposición de presidentes democráticamente electos por la vía de protestas masivas se volvió un modelo establecido de sustitución política. En esos casos extremos de alta polarización y radicalización de los principales actores, podríamos imaginar que el único resultado posible sería la caída de los presidentes, que constituiría la única salida viable para el reinicio del sistema político sobre nuevas bases, con el recrudecimiento de la polarización política y del conflicto.

No obstante, es necesario aclarar que la competencia política no siempre produce crisis, más bien al contrario. El punto ideal de la democracia pasa por la constante competencia política y la resolución de los conflictos por la vía del consenso, como por ejemplo, en la adaptación o en el surgimiento de nuevos partidos políticos que representen los temas más candentes de la sociedad. La competencia política sólo 
se vuelve perjudicial cuando ocurre de manera predatoria o extrema, pudiendo generar así inestabilidad.

Una de las principales consecuencias del descontento popular con los gobernantes en el periodo analizado puede ser percibida en los diversos intentos de reforma constitucional en el periodo. Destaco principalmente aquellas en las que se intentó avanzar el modelo democrático hacia esquemas que buscan superar las vías de la representación política tradicional expresados en los recientes modelos constitucionales discutidos actualmente en Bolivia, Ecuador y Venezuela (Pérez Flores; Cunha Filho y Coelho 2010).

Por lo tanto, la democracia en el continente, a pesar de ser relativamente joven, presenta resultados interesantes en cuanto a los anhelos de la soberanía popular. Las crisis políticas y caídas presidenciales en Argentina, Bolivia y Ecuador pueden ser analizadas, en última instancia, como representativas de la voluntad de sus ciudadanos y no como demostraciones de crisis de la democracia. Como argumenta Pérez Liñán (2003), las crisis institucionales contemporáneas en América Latina están más relacionadas a gobiernos que a la estabilidad del régimen. En la mayoría de los casos, sus orígenes estarían relacionados con una compleja interacción entre movilización social, espontánea y provocada por actores políticos, y conflictos institucionales. Samuels y Hochstetler (2011:127) indican que las consecuencias de esa nueva "inestabilidad presidencial" no son tan negativas como llega a sugerir la literatura. Al contrario, afirman que las crisis políticas que desafian el mandato de los presidentes o incluso llegan a ocasionar eventuales caídas tienden a ser relativamente cortas y sólo dejan heridas superficiales. De este modo, se ratifica la visión de quienes creen que el gran número de crisis presidenciales resueltas de manera pacífica indica, en realidad, la resiliencia de la democracia en la América Latina contemporánea.

\section{REFERENCIAS BIBLIOGRÁFICAS}

Amorim Neto, Octávio (2006). Presidencialismo e governabilidade nas Américas. Rio de Janeiro: FGV, 2006.

Amorim, Eliane; Arias, Santiane (2009). "Neoliberalismo e Lutas Sociais: a emergência dos movimentos piqueteros e altermundialistas no contexto neoliberal”. Mediações, Londrina, Vol. 14, No 2, pp. 231-251.

Anastasia, F; Ranulfo, C. \& Santos, F. (2004). Governabilidade e representação política na América do Sul. Rio de Janeiro: Fundação Konrad Adenauer/Fundação Unesp. 
Carey, John M. (2003). "Presidentialism and representative institutions" en: Domínguez, Jorge I.; Shifter, Michael (compiladores), Constructing Democratic Governance in Latin America. Baltimore:The Johns Hopkins University Press.

Chasquetti, D. (2001). "Democracia, Multipartidismo y Coaliciones en América Latina: Evaluando la Dificil Combinación”. Jorge Lanzaro (compilador). Tipos de Presidencialismo y Coaliciones Políticas en América Latina. Buenos Aires: CLACSO.

Cheibub, José Antônio (2002). "Minority Governments, Deadlock Situations, and the Survival of Presidential Democracies”. Comparative Political Studies Vol. 35, Nº , pp. 284-312.

Cheibub, J. A.; Przeworski, A.; Saiegh, S. (2004). "Government Coalitions and Legislative Success Under Parliamentarism and Presidentialism”. British Journal of Political Science, vol. $34, \mathrm{n}^{\circ} 4$, pp. 565-587.

Coutinho, Marcelo J. V. Mireles, Pedro David; Delgado, Ana Carolina (2008). "Por que protestam? Uma nova sociedade no horizonte sul-americano". Estudos e Cenários febrero. Instituto Universitário de Pesquisas do Rio de Janeiro IUPERJ/UCAM.

Dahl, Robert (1997). Poliarquia: participação e oposição. São Paulo: EDUSP.

Dávalos, P. (editor) (2005). Pueblos indígenas, Estado y democracia. Buenos Aires, CLACSO.

Downs, Anthony (1999). Uma teoria econômica da democracia. EDUSP.

Garretón, M. A. (2000). "Cambios Sociales, actores y acción colectiva en América Latina". Serie políticas sociales 56 . Santiago: CEPAL.

Fernandes, B. M. (2006). 20 anos do MST e a perspectiva da reforma agrária no governo Lula. Recuperado em 24 de setembro de 2006, de http://www2.prudente.unesp.br/dgeo/ nera/ProduçãoNERA/20anosdomsteaperspectivadareforma.pdf.

Hagopian, Frances e Mainwaring, Scott (2005). The Third wave of democratization in Latin America - advances and setbacks. New York: Cambridge University Press.

Hochstetler, Kathryn (2007). "Repensando o presidencialismo: contestações e quedas de presidentes na América do Sul”. Lua Nova, N 72, pp. 9-46.

Holfmeister, Wilhelm (2004). Reformas políticas en América Latina. Rio de Janeiro: Fundação Konrad Adenauer.

Jatobá, Daniel \& Epsteyn, Juan (2007). "A Argentina nos primeiros cinco anos do século XXI: crise, transição e transformação". En Soares de Lima, Maria Regina e Coutinho, Marcelo (org.). A agenda sul-americana: mudanças e desafios no início do século XXI. Brasília: Funag.

Jímenez Badillo, M. (2007). "Gobernando sin mayorías parlamentarias em América Latina”. Opinião Pública, Vol. 13, nº 1, pp.148-184. 
Jones, Mark (1995). Electoral Laws and the survival of presidential democracies. Notre Dame: University of Notre Dame Press.

Llanos, Mariana e Marsteintredet, Leiv (editores) (2010). Presidential Breakdowns in Latin America. Causes and Outcomes of Executive Instability in Developing Democracies. New York y London: Palgrave MacMillan.

Linz, Juan J. (1994). "Presidential or parliamentary democracy: Does it make a difference?" En: Linz, Juan J. \& Valenzuela, Arturo (editores), The failure of presidential democracy: The case of Latin America.Vol. 2. Baltimore: Johns Hopkins University Press.

Mainwaring, Scott; Brinks, Daniel \& Pérez-Liñán, Aníbal (2001). "Classificando regimes políticos na América Latina 1945 -1999”. Dados vol. 44, No 4, pp. 645-687.

Mainwaring, Scott y Scully, Thmoty (2008). "Latin America: Eight Lessons for Governance”. Journal of Democracy, Vol. 19, No 3, pp.113-127.

Melucci,A. (2001). A invenção do presente. Petrópolis:Vozes.

Mirza, Christian Adel (2006). Movimientos sociales y sistemas políticos en América Latina - la construcción de nuevas democracias. Buenos Aires: CLACSO.

Negretto, G. A. Minority Presidents and types of government in Latin America. Ponencia presentada en el mitin LASA de marzo 2003. Dallas. Texas.

O’Donnell, G. (2001). Democracia Delegativa? Novos Estudos, N³1, p. 25-40.

Pachano, Simón (2006). El proceso electoral ecuatoriano. ARI 101. http://www. realinstitutoelcano.org/analisis/1042/1042_Pachano_elecciones_Ecuador.pdf

Pérez Flores, F; Cunha Filho, C. M.; Coelho, A. L. (2010). "Participación ampliada y reforma del Estado: Mecanismos constitucionales de democracia participativa en Bolivia, Ecuador y Venezuela". Observatorio Social de América Latina,Vol. XI, N²7, pp. 73-95.

Pérez-Liñán, A. (2005). "Democratization and Constitutional Crises in Presidential Regimes: Toward Congressional Supremacy?” Comparative Political Studies Vol. 38 Nº1, pp. 51-74.

Pérez-Liñán,A. (2008). “Instituciones, coaliciones callejeras e inestabilidad política:perspectivas teóricas sobre las crisis presidenciales". América Latina Hoy, N49, pp. 105-126.

Pérez-Liñán, A. (2009). Juicio político al presidente y nueva inestabilidad política en América Latina. Buenos Aires: Fondo de Cultura Económica.

Pessanha, Charles (2002). "O poder executivo e o processo legislativo nas Constituições brasileiras - teoria e prática". En Vianna, Luiz Werneck (compliador). A democracia e os três poderes no Brasil. Rio de Janeiro: UFMG/ IUPERJ/FAPERJ. 
Postero, Nancy, G. \& Zamosc, León (eds.) (2004). The struggle for Indigenous rights in Latin America. Brighton: Sussex Academic Press.

Samuels, David y Hochstetler, Kathryn (2011). "Crisis and Rapid Reequilibration: The Consequences of Presidential Challenges and Falls in Latin America". Comparative Politics Vol. 43, N² 2, pp. 127-145.

Santos, Wanderley Guilherme (1986). Sessenta e quatro: anatomia da crise. São Paulo:Vértice.

Santos, F. G. M.;Almeida,A. S.;Vilarouca, Márcio Grijó (2008). “Um modelo de governança de esquerda com aplicação para o caso daVenezuela e o mercado comum do Sul-Mercosul”. En: Maria Regina Soares de Lima (compilador). Desempenho de governos progressitas no Cone sul: agendas alternativas ao neoliberalismo. Rio de Janeiro: Edições IUPERJ.

Segovia, Susana (2004). Indigenismo y movilización en Ecuador. En: Salvador Martí i Puig y Josep María Sanahuja (Coords.). Etnicidad, autonomía y gobernabilidad en América Latina. Salamanca: Ediciones Universidad de Salamanca.

Shugart, Matthew Soberg (1992). Presidents and assemblies: constitutional design and electoral dynamics. Cambridge: Cambridge University.

Silva, Fabricio (2011). Vitórias na crise: trajetórias das esquerdas latino-americanas contemporâneas. Rio de Janeiro: Ponteio.

Silva, Leandro Ribeiro da, yViera, Soraia Marcelino (2009). “As relações executivo-legislativo e o neoliberalismo no Brasil”. Antropolitica, No26. pp. 181-202.

Van Cott, Donna L. (2005). From movements to parties in Latin America: the evolution of ethnic politics. New York, Cambridge University.

Yashar, Deborah J. (2005). Contesting citizenship in Latin America: the rise of Indigenous Movements and the postliberal challenge. Cambridge, Cambridge University Press.

Wolff, Jonas (2007). “(De-)Mobilising the Marginalised: A Comparison of the Argentine Piqueteros and Ecuador's Indigenous Movement". Journal of Latin American Studies Vol. 39, No1, pp. 1-29.

Recibido: 20 diciembre 2011 / Aprobado: 26 abril 2012 